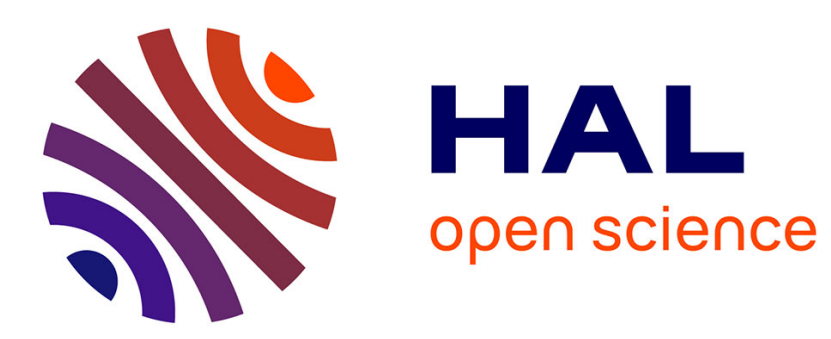

\title{
Effects of axial rectangular groove on turbulent Taylor-Couette flow from analysis of experimental data
}

K. Sodjavi, Florent Ravelet, F. Bakir

\section{To cite this version:}

K. Sodjavi, Florent Ravelet, F. Bakir. Effects of axial rectangular groove on turbulent TaylorCouette flow from analysis of experimental data. Experimental Thermal and Fluid Science, 2018, 10.1016/j.expthermflusci.2018.04.022 . hal-01611013v2

\section{HAL Id: hal-01611013 \\ https://hal.science/hal-01611013v2}

Submitted on 26 Feb 2018

HAL is a multi-disciplinary open access archive for the deposit and dissemination of scientific research documents, whether they are published or not. The documents may come from teaching and research institutions in France or abroad, or from public or private research centers.
L'archive ouverte pluridisciplinaire HAL, est destinée au dépôt et à la diffusion de documents scientifiques de niveau recherche, publiés ou non, émanant des établissements d'enseignement et de recherche français ou étrangers, des laboratoires publics ou privés. 


\title{
Effects of axial rectangular groove on turbulent Taylor-Couette flow from analysis of experimental data
}

\author{
K. Sodjavi ${ }^{\mathrm{a}}$, F. Ravelet ${ }^{\mathrm{a}, *}$, F. Bakir ${ }^{\mathrm{a}}$ \\ ${ }^{a}$ Arts et Metiers ParisTech, DynFluid, 151 boulevard de l'Hpital, 75013 Paris, France
}

\begin{abstract}
The effects of the number of surface grooves on the hydraulic resistance are experimentally investigated in the case of fully turbulent Taylor-Couette flow at a fixed radius ratio $\eta=0.9375$ and for Reynolds numbers $\operatorname{Re}_{i}$ from $2 \times 10^{4}$ to $13 \times 10^{4}$. Three configurations of inner cylinder having 6,12 and 24 grooves of the same rectangular shape and regularly distributed are compared to a reference smooth cylinder case. Such configurations are common in electric motors of high power density and of very high rotation rate, for which better understanding and knowledge of the flow patterns and aerodynamic losses are essential to enhance their design and to develop appropriate cooling systems. Torque and pointwise velocity measurements are performed. The effects of the flow modulations induced by the grooves on the friction coefficient and the flow features are examined. The results show significant difference in the average flow pattern, the presence of the grooves suppressing the Taylor Vortices. An increase of the friction coefficient with the groove number is reported. However, the individual drag-increasing contribution of each groove is reduced when increasing the groove number. It is also found that the local scaling exponent of the friction coefficient as a function of the Reynolds number is insensitive to the present surface roughness, suggesting that the bulk flow contribution is similar for all the four configurations.
\end{abstract}

Keywords: Rough boundary, Rectangular groove, Friction coefficient, Taylor-Couette flow, LDV measurement,

\section{Introduction}

Fluid flow around solid body of rough or structured surface topology has attracted considerable interest since it is encountered in many natural phenomena and engineering applications. The design of more compact electric motor with high power density is one of the related application. Generally, this configuration is analogous to a canonical topology known as Taylor-Couette flow, consisting of the flow between two coaxial cylinders with a stationary outer cylinder and a rotating inner cylinder. In some cases an axial flow is also considered and the flow is then refered to as Taylor-Couette-Poiseuille flow. As noticed by Fénot et al. [1 in their review, there are a lot of studies and results for the aerodynamics and heat transfer capacity on smooth cylinders, but the few studies dedicated to slotted cylinders mainly focused on the heat transfer. For the specific issue of high power compact electric motors rotating at very high rotation rates, with rotors presenting slotted shapes for electromagnetical reasons, the aerodynamic losses in the annulus between the two cylinders become a major issue to optimize their performances. Better understanding of the flow field and to a lesser extent of the heat transfer characteristics in this confined environment still requires dedicated studies for

\footnotetext{
* Corresponding author

Email address: florent.ravelet@ensta.org (F. Ravelet)
}

axially grooved inner cylinders. This is needed in order to enhance their design and to develop appropriate cooling system. As far as heat and aerodynamic behavior are concerned only a few investigations contain references to an axially grooved cylinder. Likewise, most of the reported results concern global physical quantities.

For instance, Hayase et al. 2 used numerical simulation to compare three different flow configurations in terms of momentum and heat transfer coefficient in a laminar flow. The authors specifically studied the influence of twelve cavities embedded in either the inner rotating or the outer fixed cylinder in laminar flow. They observed larger effects, both on the momentum and heat transfer when the cavities were located on the inner cylinder rather than on the outer cylinder. Lee \& Minkowycz [3] and Gardiner \& Sabersky [4] investigated experimentally the heat transfer and pressure drop characteristics of smooth or axially grooved cylinders, with a superimposed Poiseuille flow, for Reynolds numbers based on the rotation rate and on the gap width up to $R e_{i} \simeq 4000$. The authors observed that the presence of grooves on the rotating inner cylinder significantly increased the heat coefficient. They also noted that the grooved rotor were associated to friction factors which were generally higher than those for a smooth rotor.

Several experimental studies, such as, for instance that of Cadot et al. [5], van den Berg et al. [6], or Motozawa et al. [7] were devoted to the dissipation in Taylor-Couette 
flows with regularly distributed square or triangular ribs on the surface of the cylinders, at much higher Reynolds numbers (up to $R e_{i}=3 \times 10^{5}$ ). The main conclusions in Refs. [5, 6] is that with two rough cylinders the drag coefficient is independent of the Reynolds number. The authors observed that the total dissipation is dominated by the contribution of the boundary layers for smooth cylinders, whereas it is dominated by the bulk flow contribution for ribbed cylinders. In addition to these experimental studies, some numerical studies such as that of Tsukahara et al. 8] have considered the effect of ribs on the global transport properties and flow characteristics for Reynolds number as large as $3.2 \times 10^{3}$. The most important conclusion drawn from the research was that the increment in the pressure drag due to the roughness is damped as a result of the strong adverse pressure gradient behind each rib. In Refs. [7, 8, the distance between two consecutive grooves $(\lambda)$ was used as a key parameter and its influence on the mean flow and the friction coefficient was highlithed.

All the above cited studies were performed with structural rough elements arranged in such a way as to obstruct the mean flow. This type of surface roughness is used either as heat transfer promoters or results from design necessity. They are known to be more efficient in generating friction drag than smooth surface. Rough elements aligned with mean flow is another category of surface roughness generally used to reduce the skin friction drag. This is achieved when the boundary layer thickness becomes smaller than the rough height and leading the flow resistance to be bulk dominant. The studies of Hall \& Joseph [9], Moradi \& Floryan [10, Greidanus et al. 11] and Zhu et al. [12] can be include in this category.

A better understanding of the relevant mechanisms associated with an increase of friction drag in axially grooved cylinder flow involves a full understanding of the TaylorCouette flow. Since the pioneering contribution of Couette [13], the Taylor-Couette flow has been greatly studied, both experimentally, numerically and theoretically because of its considerable scientific and engineering importance. The recent comprehensive review of Grossmann et al. 14 provides salient details on its fully developed turbulence regime from experimental, numerical, and theoretical points of view.

In this paper, we focus on the statistical features of fully turbulent Taylor-Couette flow with an axially grooved inner rotating cylinder. The grooves have the same rectangular shape and are regularly distributed on the tested cylinders. In an attempt to investigate the effect of the groove density, we conduct series of experiments using four different configurations. Three configurations of inner cylinder having 6,12 and 24 grooves of the same rectangular shape and equally spaced in azimuthal direction are compared to a reference smooth cylinder. For each configuration, torque and pointwise velocity measurements are performed to assess the global transport properties and flow characteristics. Section 2 describes the experimental setup, the geometry of each tested cylinder and the measurement techniques. The results are then analysed in section 3. The characteristics of the mean flow are summarized in section 3.1. with a focus on the noticeable effects of the groove density. The local flow characteristics are analysed in section 3.2 , with a focus on the interaction of the groove and bulk flows. The torque results expressed in terms of friction coefficient are discussed in section 3.3 . Then, concluding remarks are given in section 3 .

\section{Experimental setup}

\subsection{Taylor-Couette facility}

The experimental apparatus has been specifically designed for the study of the Taylor-Couette flow. A detailed sketch and Photograph of this facility are given in Fig. 1. It consists of two coaxial cylinders that create a gap occupied by a working fluid. Each cylinder can rotate independently with the use of an AC Servo motor (Panasonic Minas A5, $1500 \mathrm{~W})$. The outer cylinder made of $5 \mathrm{~mm}$ thick Plexiglas is driven via a driving belt while the inner cylinder is directely attached to a shaft through the torque sensor. The system is closed at the top and bottom ends with lids that rotate with the outer cylinder. The motors are controlled by a LabView software program that regulates the desired angular rotation rate of the corresponding cylinder. The radii of inner and outer cylinders are $r_{i}=112.5 \mathrm{~mm}$ and $r_{o}=120 \mathrm{~mm}$, respectively, yielding to a gap $d=r_{o}-r_{i}=7.5 \mathrm{~mm}$ and to a gap ratio of $\eta=r_{i} / r_{o}=0.9375$. All the tested inner cylinders have a length $h=200 \mathrm{~mm}$, which gives an axial aspect ratio of $h / d=26.66$. The axial gaps between the top and bottom end-plates of the inner and outer cylinders are $2.5 \pm 0.1 \mathrm{~mm}$. All the results of this study concerns rotor/stator configurations, therefore the outer cylinder was kept at rest mechanically while varing the inner cylinder angular frequency $\omega_{i}$. The relevant dimensionless parameter that characterizes the flow dynamic is the inner Reynolds number defined as:

$$
R e_{i}=\frac{r_{i} \omega_{i} d}{\nu}
$$

\subsection{Geometry of the inner cylinders}

Our aim is to evaluate the action of axial grooves on the behaviour of Taylor-Couette flow, more precisely to identify possible mechanisms responsible for the increase in flow resistance. Thus three grooved inner cylinders are compared to a reference one having a smooth surface. Please note that for all the tested cases, the outer cylinder has a smooth surface.

Figure 2 presents the four inner-cylinder configurations. As can be seen, the grooved cylinders are equipped with 6, 12 or 24 identical axial rectangular cavities which are arranged periodically. They are named " $G n$ " where $n$ is the number of grooves. The detailed shape of the groove is shown at the bottom of Fig. 2. The ratio of the groove width $w=12 \mathrm{~mm}$ to its depth $k=5.84 \mathrm{~mm}$ is close 

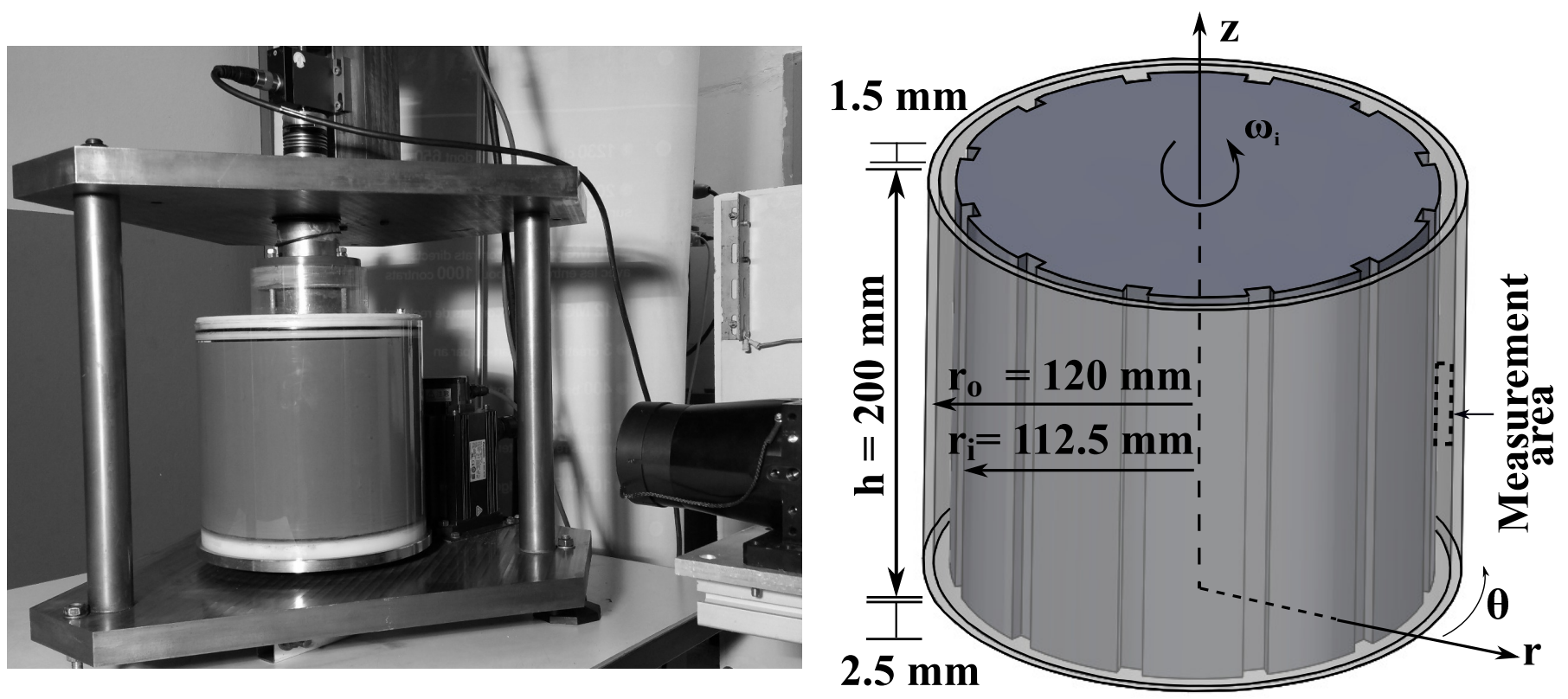

Figure 1: Photograph and sketch with dimensions of the experimental setup. Left: Taylor-Couette facility with torque meter at upper part of the picture and laser head at left side. Right: sketch showing the grooves of the inner cylinder and LDV Measurement area

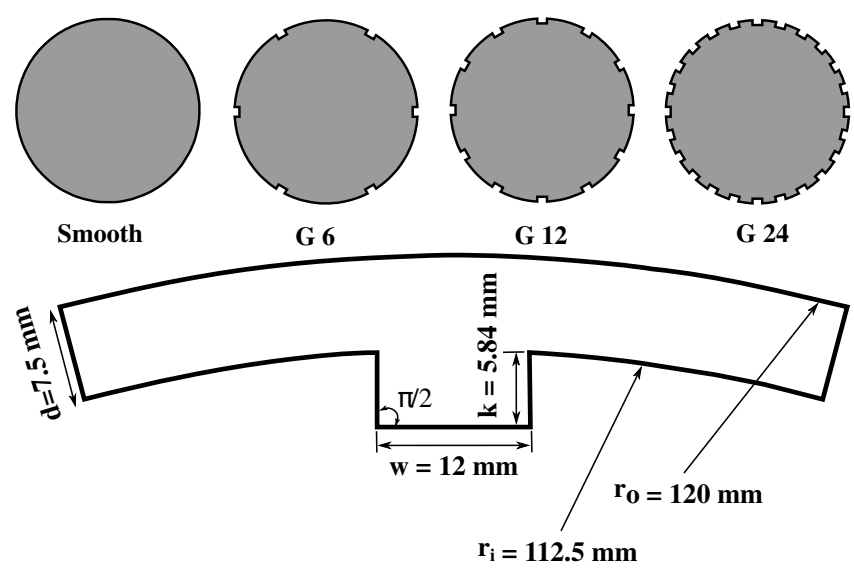

Figure 2: Schematic top view of the tested inner cylinders and the groove geometry. (Top), left to right reference smooth cylinder, cylinder with six $(G 6)$, twelve $(G 12)$ and twenty four $(G 24)$ grooves arranged at uniform spacing $\lambda=2 \pi r_{i} / n ; n=6,12,24$. (Bottom) Dimensions of elementary groove to $w / k \simeq 2$. These parameters are motivated by the intended application, namely the investigation of aerodynamic losses in an electric motor. The ratio of the groove separation distance $\lambda=2 \pi r_{i} / n$ to the groove depth $k$ is generally used as the key parameter to distinguish the different wall roughness configurations [7, 8]. In the present work, this ratio $\lambda / k$ corresponds approximately to 20,10 and 5 for $G 6, G 12$ and $G 24$ respectively.

\subsection{Torque measurement}

The torque $(T)$ is measured on the inner cylinder using a co-rotating torque meter (KISTLER Ref.4503A, 10 N.m) with an absolute precision of less than \pm 0.02 N.m. As the torque meter is mounted on the shaft between the driving motor and the inner cylinder, the measured torque includes the mechanical friction of the bearings. This contribution is measured in case of air filled flow as a function of the rotation rate and is subtracted from the total torque via a linear interpolation. This contribution is less than $2 \%$ of the torque for all the runs. The present TaylorCouette system is operated with water as working fluid at an initial temperature in the range $20-24{ }^{\circ} \mathrm{C}$, resulting in a volumetric mass density of the order of $\rho=998 \mathrm{~kg} \cdot \mathrm{m}^{-3}$ and a kinematic viscosity of the order of $\nu=10^{-6} \mathrm{~m}^{2} \cdot \mathrm{s}^{-1}$. The system is not equipped with temperature regulation unit and therefore the mean temperature increases during a run, resulting in a variation of the viscosity and density. a K-type thermocouple flush mounted under the top lid, in direct contact with the working fluid, is used to measure the temperature rise due to frictional heating. For each run, the rotating frequency of inner cylinder is increased stepwise from 3.5 to $20 \mathrm{~Hz}$ at constant acceleration rate, $\partial R e_{i} / \partial \tau=149102$ where $\tau$ is a non-dimensional time de- 
fined as $\tau=t \nu / d^{2}$. During each step, once the desired angular frequency is achieved, the torque, the angular frequency and the working fluid temperature are recorded simultaneously at a sampling frequency of $5 \mathrm{kHz}$ during $40 \mathrm{~s}$. The experiments are repeated three times for each inner cylinder over the same Reynolds number range to check the reproducibility. The repeatability is quite satisfactory, the variation being typically less than $2.5 \%$. The measured torque is expressed in term of friction coefficient as follows:

$$
C_{f}=\frac{T}{2 \pi \rho r_{i}^{2} h\left(r_{i} \omega_{i}\right)^{2}}
$$

In the two horizontal gaps between the inner cylinder and the top or bottom lid, the fluid motion creates additional shear force. To estimate this contribution and subtract it from $C_{f}$, we use the empirical correlation developed by Hudson \& Eibeck 15] in turbulent flow with single rotating disk and different shroud clearance ratios and gap aspect ratios. The torque that is applied on the disks $T_{\text {disk }}$ is expressed as follows:

$$
T_{d i s k}=\frac{1}{2} C_{d i s k} \rho \omega_{i}^{2} r_{i}^{5}
$$

with $C_{\text {disk }}=2.83\left(r_{i}^{2} \omega / \nu\right)^{-0.48}\left(g / r_{i}\right)^{0.05}$. Here, $g$ is the axial height of the gap between the top or bottom endplates of the inner and outer cylinders. In the present case, the relative contribution of the end-effects to the measured torque is estimated to be in the ranges $9-17 \%, 7-12 \%$, $6-11 \%$ and $4-8 \%$ for, respectively, the smooth, G6, G12 and $G 24$ cylinders.

\subsection{LDV measurement}

The spatial distribution of the mean velocity in the meridional plane of the gap between the two cylinders (see Fig. 1) is measured by single-component Laser Doppler Anemometry (LDA), using a Dantec Dynamics FlowExplorer system, in a backscattering configuration. It consists of a $25 \mathrm{~mW}$ argon-ion laser with red wavelength $(660 \mathrm{~nm})$, and a fiber-optics probe of focal length $300 \mathrm{~mm}$ giving a measurement volume of $0.1 \times 0.1 \times 1 \mathrm{~mm}^{3}$. The sytem has a high accuracy, as the calibration coefficient uncertainty is lower than $0.1 \%$. The working fluid is seeded with iriodin 201 of $7-14 \mu \mathrm{m}$ mean diameter used as the tracer particles. For each inner cylinder, azimuthal and axial velocities are measured at 585 points distributed along 39 radial profiles at the mid-height of the rotational axis (see left part in Fig. 1) at constant axial intervals of $1 \mathrm{~mm}$. At each measurement position, 100000 samples are acquired, with a limiting time acquisition of $40 \mathrm{~s}$. The data rate lies in the range $800-10000 \mathrm{~Hz}$, while the validation rate is upper than $60 \%$. In dealing with velocity measurements in liquid Taylor-Couette flow by mean of LDA technique, the presence of flat or curved interface introduces a shift of the measurement volume and an underestimation of some components of the flow velocity (Huisman et al. [16]). In this study a ray tracer program has been used to calculate the measurement volume displacement and velocity correction factors. The velocity correction factor is only applied to the azimuthal velocity, because for the axial velocity the laser beams are normal to the flat plate and therefore the difference in refractive index is balanced by the change in wavelength.

\section{Results and discussion}

\subsection{Mean flow characteristics}
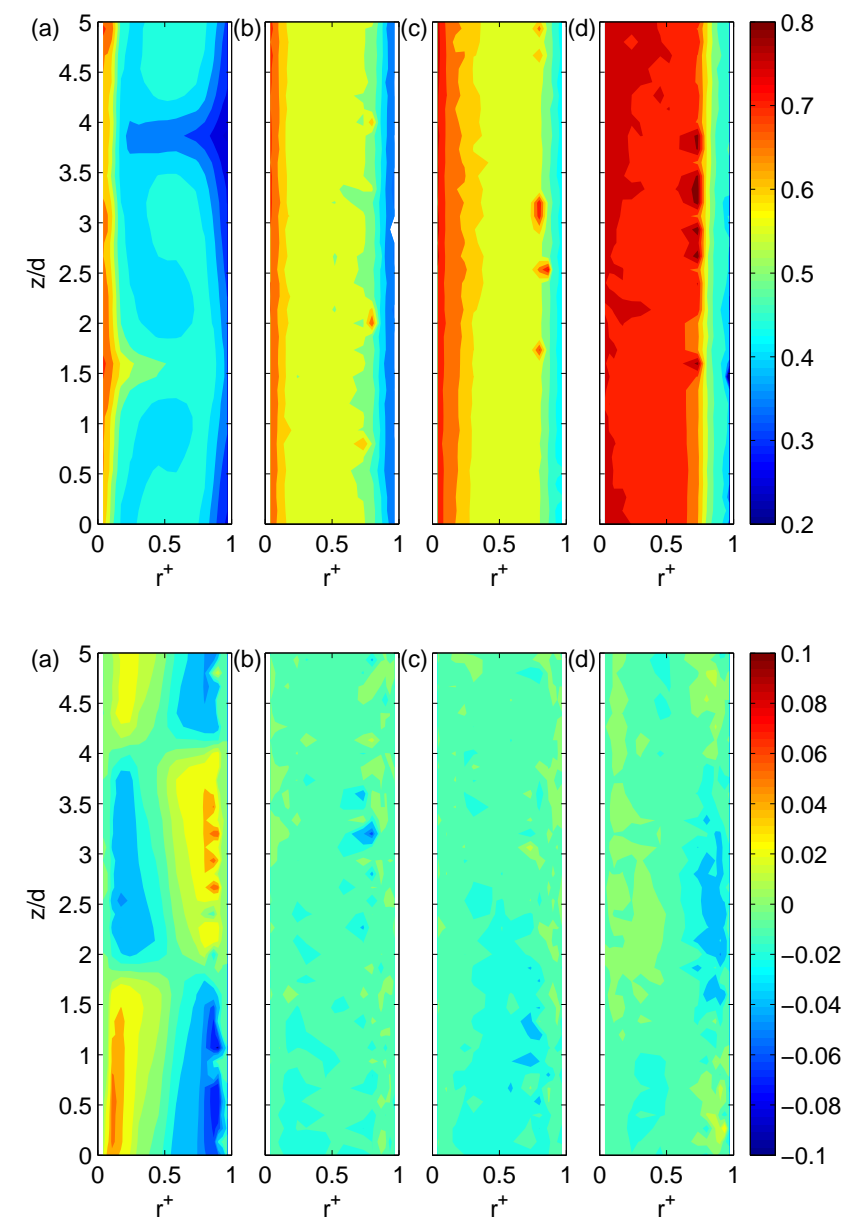

Figure 3: (Colour online) Contour plots of the time-averaged normalized azimuthal (top) and axial (bottom) velocity fields $V_{\theta}$ measured by LDA at Reynolds number $R e_{i}=47000$. (a) Smooth case, (b) G6 case, (c) G12 case, and (d) G24 case

Prior to examining the friction coefficient, we investigate the effect of groove on the overall behaviour of the bulk flow. To characterize the turbulent states in the grooved and smooth cases, we perform scans of the azimuthal and axial velocities in the meridional plane $(r, z)$ at mid-height of the apparatus for two Reynolds number $R e_{i}=47000$ and 80000 . At these Reynolds numbers, the flow is considered as fully turbulent. The dimensionless radial coordinate is $r^{+}=\left(r-r_{i}\right) / d$ so that the inner cylinder 
is at $r^{+}=0$ while the outer cylinder is at $r^{+}=1$. The wall inner cylinder velocity $r_{i} \omega_{i}$ is used as velocity scale.

Figure 3 compares the time-averaged azimuthal and axial velocity contour plots for $R e_{i}=47000$, showing the flow characteristics for the four different inner cylinder surfaces. In the case of the smooth surface, the presence of large-scale structures is clearly highlighted by the radial inflow and outflow boundaries visible on the azimuthal velocity field (see top part in Fig. 3(a)). These structures are also visible through the alternation of positive and negative velocity visible on the axial velocity field (see bottom part in Fig. 3 (a)). From these boundaries, high-speed fluid near the inner cylinder and low-speed fluid near the outer cylinder are ejected in the bulk regions between the vortices and contribute to redistribute the angular momentum. The elliptical shape of these vortices depends on the strength of inward and outward fluid. According to Tokgoz et al. 16 the birth and death of new vortices always takes place at these boundaries. For the present geometry, Reynolds number and acceleration rate $\left(\partial R e_{i} / \partial \tau\right)$, the local axial wavelength of the vortices obtained at midheight of the apparatus is around $4.4 d$, comparable to the value reported by Ravelet et al. [17] for $R e_{i}=14000$ in an experiment with a slightly different gap ratio.

The three grooved cylinder results reveal certain constants in the behavior of the velocity fields and notable structural differences compared to smooth surface case. Contrary to the smooth cylinder, no axial periodicity is observed indicating the absence of large coherent structures. As expected, the grooves produce radial velocity modulations and the resulting interaction with the bulk flow breaks the primary instability responsible of CouetteTaylor roll formation. The random distribution of the axial velocity confirms the breakdown of coherence and the small scale structures of the flows. Also from the axial velocity field, one can state that there is negligible convective transport or diffusion of azimuthal momentum in the bulk flow. From comparison between the grooved surface cases, one can notice that the region of high azimuthal velocity near the inner cylinder gradually extends into the bulk flow with increasing groove number (see top part in Fig. 3 (bd)) and that the azimuthal velocity becomes more uniformly distributed along the axial direction. The change in magnitude of azimuthal velocty in the bulk is not fully understood. However, in our opinion, it may result from ejection of high-speed fluid at the top edges of each groove. In fact, the flow separation occuring at the top edges of the grooves may generate a radial high-speed fluid ejection towards the outer cylinder. As the groove number increases, the contribution of the ejected fluid will increase, explaining the changes observed in magnitude of the azimuthal velocity contours.

In order to check the dependence of the flow structure on the Reynolds number, Fig. 4 compares four timeaveraged azimuthal and axial velocity contour plots at $R e_{i}=80000$. At this Reynolds number, the average flow with grooved wall shares common features with those at
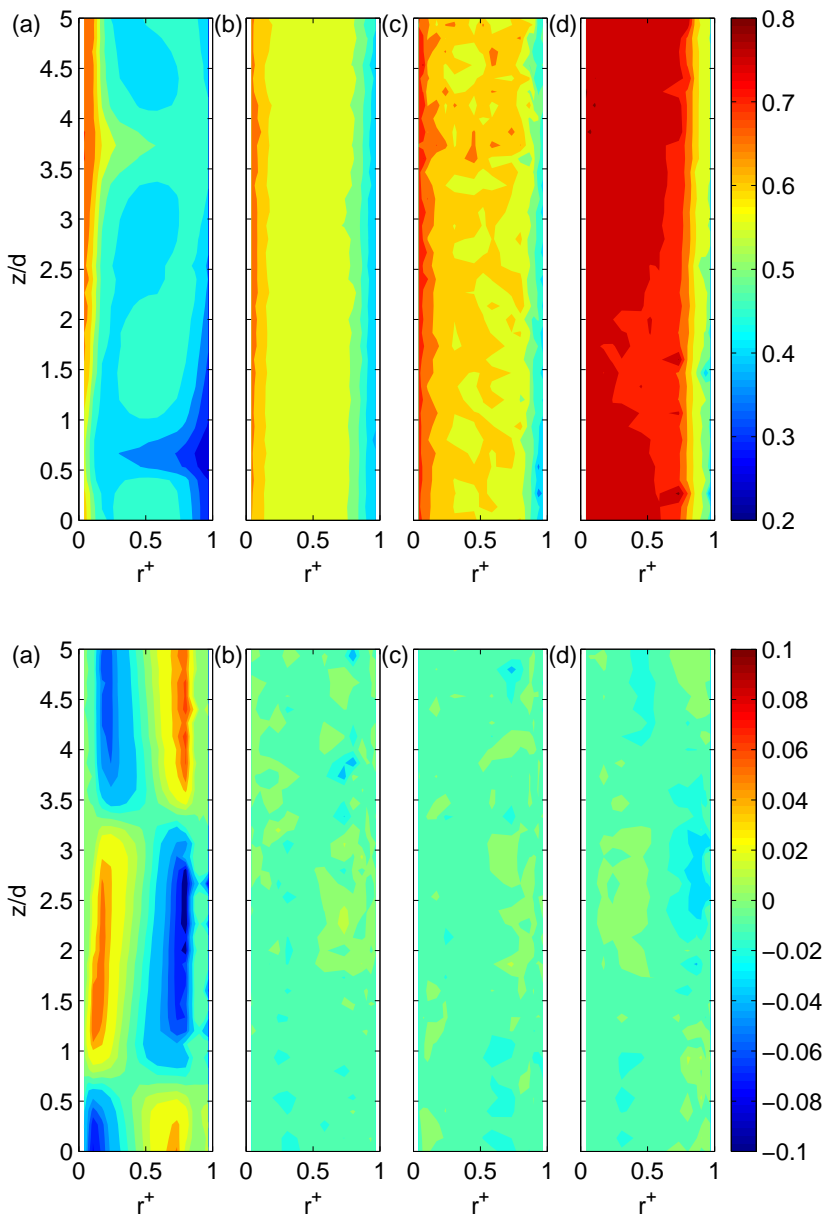

Figure 4: (Colour online) Contour plots of the time-averaged normalized azimuthal (top) and axial (bottom) velocity fields $V_{\theta}$ measured by LDA at Reynolds number $R e_{i}=80000$. (a) Smooth case, (b) $G 6$ case, (c) $G 12$ case, and (d) G24 case 
$R e_{i}=47000$, such as the loss of axial periodicity and the increase in magnitude of azimuthal velocity in the bulk as the groove number increases. The only noticeable difference is a larger axial wavelength of around $6 d$ obtained with the smooth surface case. Based on these results, we can rigorously claim that this specific axial groove geometry enhances the momentum transport in the radial direction, i.e. from the inner cylinder to the bulk flow at high Reynolds number.
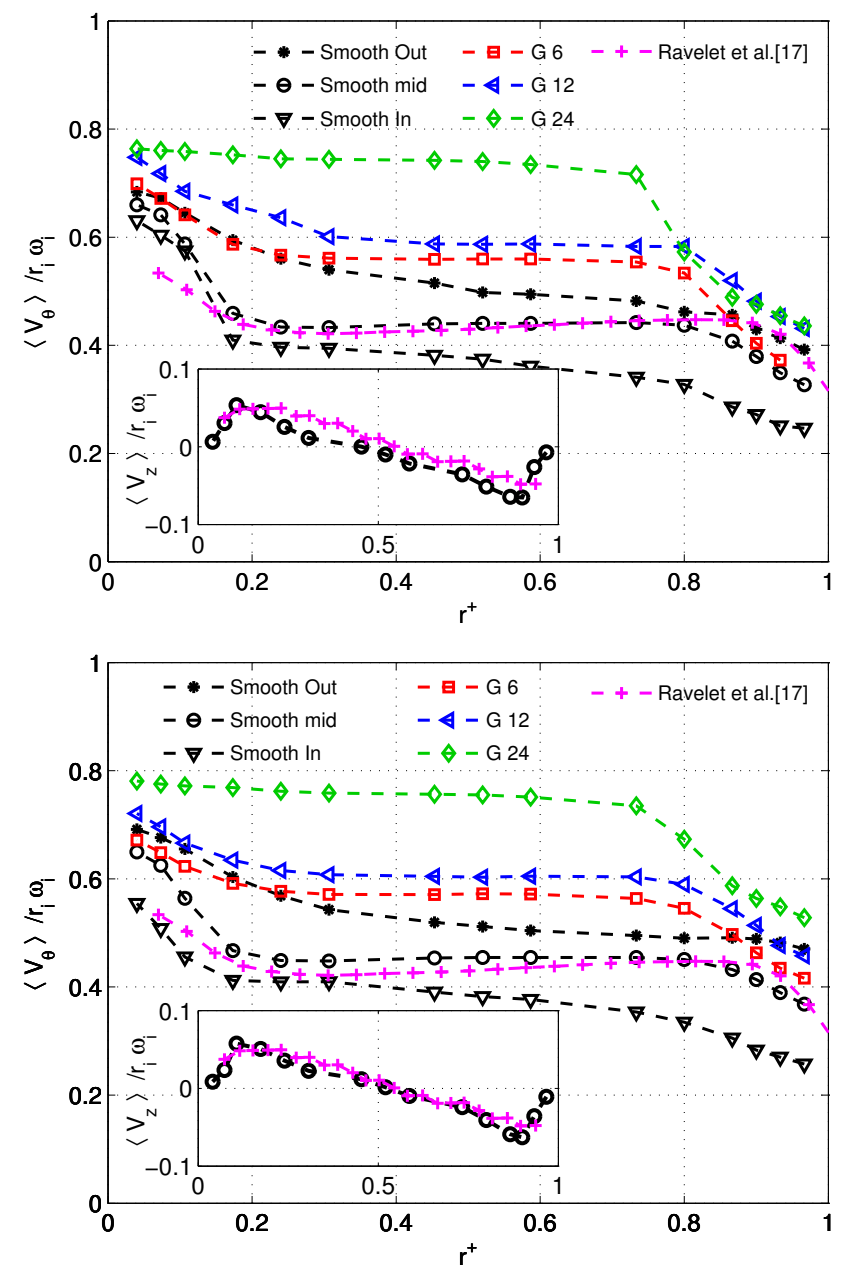

Figure 5: Normalized averaged azimuthal $\left\langle V_{\theta}\right\rangle$ and axial $\left\langle V_{z}\right\rangle$ velocity profile along radial coordinate for Reynolds number $R e_{i}=47000$ (top) and $R e_{i}=80000$ (bottom). The three grooved cylinder profiles have been averaged over both time and axial position. The smooth surface profiles were extracted at, the center of a vortex (labelled Smooth mid), intflow (labelled Smooth In) and outflow (labelled Smooth Out) boundaries between two consecutive vortex. The subfigure displays the axial velocity profile extracted at the center of a vortex. Comparison to the data of Ravelet et al. [17.

In Fig. 5 we compare profiles of the mean azimuthal velocity across the gap between the cylinders for the two Reynolds numbers. The three grooved cylinder profiles have been averaged along the axial position. The smooth surface profiles are extracted at three relevant axial locations: at the center of a vortex, at the inward and at the outward vortex boundaries. The data of Ravelet et al. [17] extracted at the center of a vortex are added for comparison. Globally, the overall trend of the present profiles is comparable to the results reported by Ravelet et al. [17. The slight gap observed on the azimuthal velocity profile is probably due to the different value of the Reynolds number or to a gap ratio dependence. A strong signature of the number of grooves can be seen on these profiles as mentioned previously. As the groove number increases, the profiles become more flat near the rotating cylinder, leading to a steeper velocity gradient. This corresponds to the so-called downward shifts in the log-law region, as it is well established that any rough surface leads to a downward shift in the logarithmic region of the velocity profile ([18, 19]). The increase in the azimuthal velocity, averaged axially at mid-gap is constant for the two Reynolds numbers and is approximately 27\%, 33\% and $68 \%$ of the smooth case value for $G 6, G 12$ and $G 24$, respectively. An increase in bulk azimuthal velocity in the presence of wall roughness has also been reported from experimental data by Greidanus et al. 11 and from numerical data by Hayase et al. [2] and Zhu et al. [12. The latter authors attributed it to the conservation of the angular velocity current along the radius.

To gain some insight on the velocity fluctuations, the root mean square azimuthal velocity profiles extracted at mid-gap in the bulk flow are plotted against the axial coordinate in Fig. 6 for Reynolds number $R e_{i}=47000$. We do not include the profiles for $R e_{i}=80000$ because they are identical to those at $R e_{i}=47000$. As expected the grooves do not only impact the mean flow, but also the fluctuations. The three grooved cylinders exhibit nearly constant value along the axial direction, with a slightly higher intensity for G24. For the smooth cylinder, the profile exhibits a prominent double-peak feature characteristic of the inflow and outflow boundaries of Taylor vortice. Its mean intensity is lower than those of the grooved cylinders. Globally, such behaviors are consistent with those of mean flow and could be attributed to competing factors of groove-induced modulations that add periodically intermittency or heterogeneity in bulk flow.

\subsection{Local flow characteristics}

In order to perform spectral analysis of the flow unsteadiness, time-resolved signals of the azimuthal velocity have been recorded at a mean acquisition frequency of around $10 \mathrm{kHz}$ and maximum point time series of 1000000 . These parameters correspond to approximately 1132 and 662 points per revolution of the inner cylinder for $R e_{i}=$ 47000 and $R e_{i}=80000$, respectively. Before computing Fourier transform, all LDV signals are reconstructed on an equally spaced time grid based on local mean sampling frequency via zeroth-order interpolation. All the power spectra are estimated using the periodogram technique with a Hanning window and an overlapping of 50\%. Each spectrum is expressed in decibels $(\mathrm{dB})$ using the following 


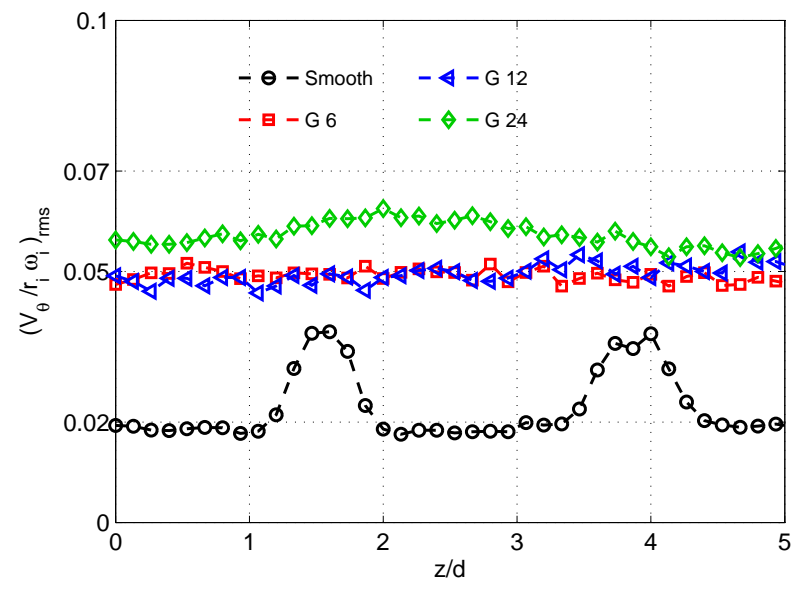

Figure 6: Normalized root mean square azimuthal velocity profile along axial coordinate for Reynolds number $R e_{i}=47000$. The profiles have been extracted at $r^{+}=0.5$.

formula:

$$
S(f)=20 \log _{10}\left(p(f) / p_{0}\right)
$$

where $p(f)$ is the power spectrum density function and $p_{0}$ is taken equal to unity. The frequency is normalized by the groove passing frequency $f_{g p f}=n \omega_{i} / 2 \pi$.

Figures 7 and 8 compare the power spectra of the azimuthal velocity fluctuation at three measured points distributed across the flow, one in the bulk flow and two others near the inner and outer cylinder boundary layers. These figures help observing the flow dynamics in more details. Near the innner cylinder at $r^{+}=0.1$, for both Reynolds numbers, the spectra exhibit seven strong discrete peaks, illustrating the complexity of the near-wall flow. The first peak, at $f=f_{g p f}$, is associated with the groove passing frequency, whereas the others correspond to its harmonics. At this location, the distribution of turbulent energy between the different harmonics depends on the groove number. The energy content for G6 is quasiconstant for all the harmonics (see Figs. 7 (a) and 8 (a)), while it gradually attenuates for $G 12$ and $G 24$. We relates it to the distance between the grooves, not sufficient for the flow to be recovered, so leading to complex unstable flow. The fact that the intensity of the base frequency and its harmonics declines quicker with more grooves could also been related to the fact that with more grooves the differences in velocity between the forcing inner cylinder and the bulk velocity decreases: the forcing per groove decreases, as also seen later when analyzing the contribution to the torque. In the bulk flow at $r^{+}=0.55$, the influence of the groove becomes slightly weaker. Apart from the groove passing frequency, the spectra display approximately three, one and zero low energy peaks (see Figs. 7 (b) and 8 (b)) for $G 6, G 12$ and $G 24$, respectively. This is consistent with observations at $r^{+}=0.1$ about the dependence of the groove signature on its number. With a
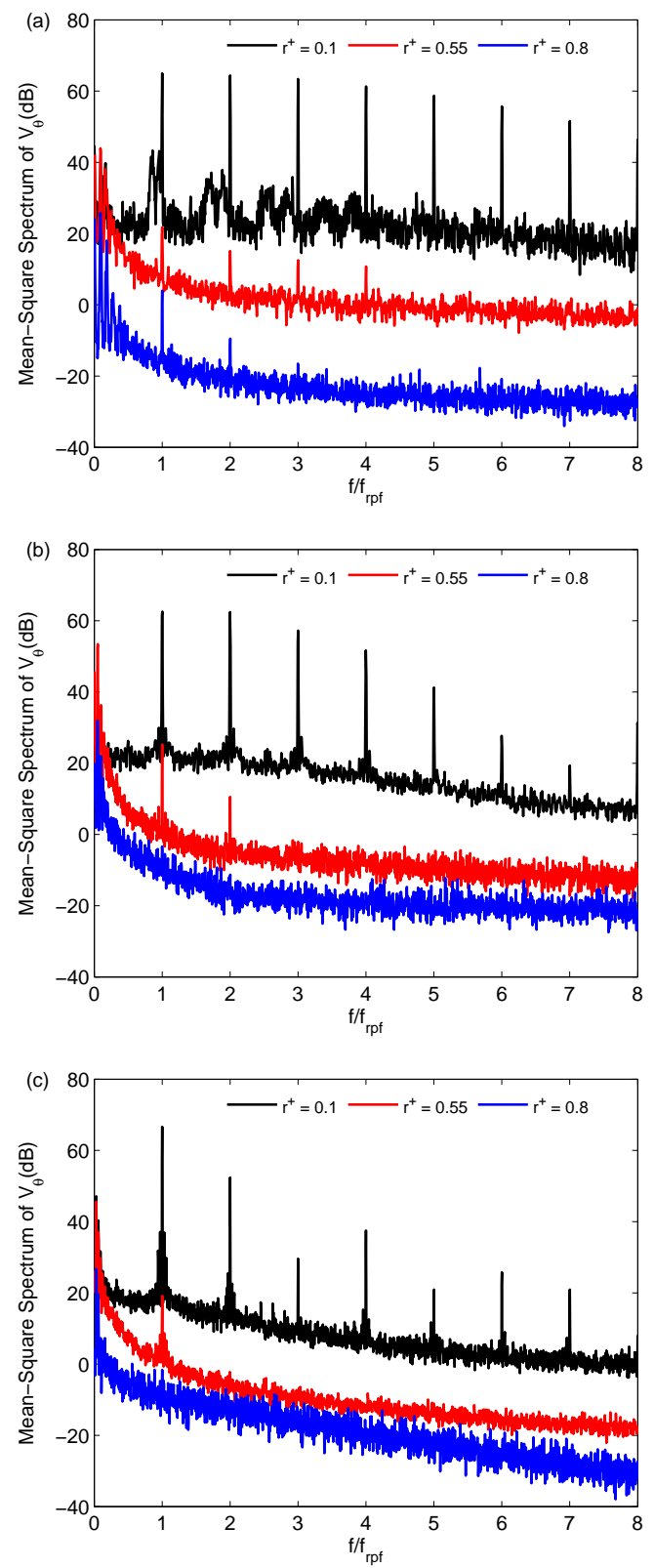

Figure 7: Square amplitude spectra of tangential velocity $V_{\theta}(t)$ at three different locations for Reynolds number $R e_{i}=47000$. The first and the third spectrum is shifted $13.98 \mathrm{~dB}$ upward and $-26 \mathrm{~dB}$ downward with respect to the middle one, respectively. (a) $G 6$ case, (b) G12 case, and (c) G24 case 

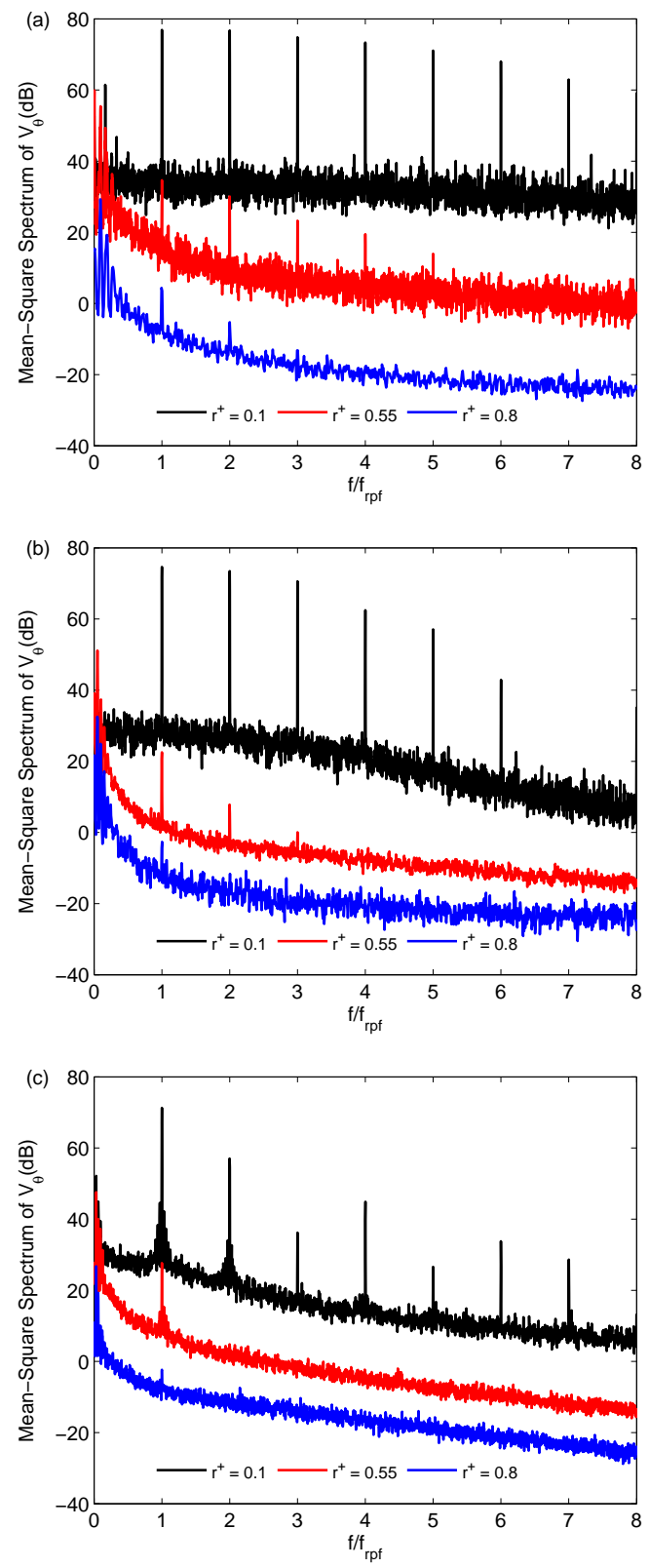

Figure 8: Square amplitude spectra of tangential velocity $V_{\theta}(t)$ at three different locations for Reynolds number $R e_{i}=80000$. The first and the third spectrum is shifted $13.98 \mathrm{~dB}$ upward and $-26 \mathrm{~dB}$ downward with respect to the middle one, respectively. (a) $G 6$ case, (b) $G 12$ case, and (c) $G 24$ case deeper penetration, at $r^{+}=0.8$, the energy content of the peak is gradually diminished and the peaks are completely suppressed for G12 and G24 (see Figs.7(c) and 8(c)). For the two cases, the disappearance of the sharp peaks does not imply necessarily that the flow is totally featureless in this region. The large value of $\lambda$ - the distance between two consecutive grooves - ensures a low interaction between consecutive groove modulated waves, probably responsible for the persistence of the peak in $G 6$ flow.

Flow characteristic analysis based on spectra indicates different local dynamics in relation to groove number and spatial location. In the following, we propose to go further in perfoming spatial analysis using phase-average technique. The LDV signals reconstructed via zeroth-order interpolation at mean sampling frequency $f_{a c q}$ are decomposed into $N$ (integer number) revolutions of period $\tau=$ $2 \pi / \omega_{i}$. Each period contains $K=2 \pi f_{a c q} / \omega_{i}$ samples, where $K$ is an integer. From the velocity phase-averaged at $\phi=j \omega_{i} / f_{a c q}(j=1, \ldots, K)$, we define the mean (Eq. 5) and standard deviation (Eq. 6) of azimuthal velocity:

$$
\begin{aligned}
& \left\langle V_{\theta}(\phi)\right\rangle=\frac{1}{N} \sum_{i=0}^{N-1} V_{\theta}(\phi+i \tau), \phi \in[0,360]^{o}, \\
& R M S\left\langle V_{\theta}(\phi)\right\rangle=\frac{1}{N} \sum_{i=0}^{N-1} V_{\theta}(\phi+i \tau),
\end{aligned}
$$

In Fig. 9, we compare profiles of the phase-averaged azimuthal velocity normalized by the rotation velocity of the inner cylinder wall. Only the case $R e_{i}=47000$ is shown since the distribution is identical for the two Reynolds numbers. The signature of the groove can be observed in both near-wall and bulk flow locations. Near the rotating wall at $r^{+}=0.1$, the profiles of mean azimuthal velocity are highly periodic and regular with only a single dominant frequency corresponding to groove passage. The periodicity is $60^{\circ}, 30^{\circ}$ and $15^{\circ}$ for $G 6, G 12$, and $G 24$, respectively. The profiles also show that the mean flow near the inner cylinder is dominated by the velocity deficit created by the groove passages. This can be explained by the presence of the steady recirculation flow in the groove. In fact, literature data based on the channels flow with rectangular cavities [18, 19] or Taylor-Couette flow with grooved inner or outer cylinder [2] revealed a recirculating flow within the cavities. The presence of this recirculating flow will induced by viscous force a shear zone at each groove passage, which will result in the azimuthal velocity deficit. Another interesting observation revealed by phase-averaged technique is the higher fluctuation level within the velocity deficit region. This observation is a consequence of a higher shear stress and velocity gradient induced by the cavity shear layer which generate an intense turbulent production. The phase-averaged profiles in the bulk flow at $r^{+}=0.55$ show weakness of groove signatures as compared to $r^{+}=0.1$. The fluctuation level is more attenuated and its maximum location is shifted to center of the crest, contrary to what happens at $r^{+}=0.1$. 

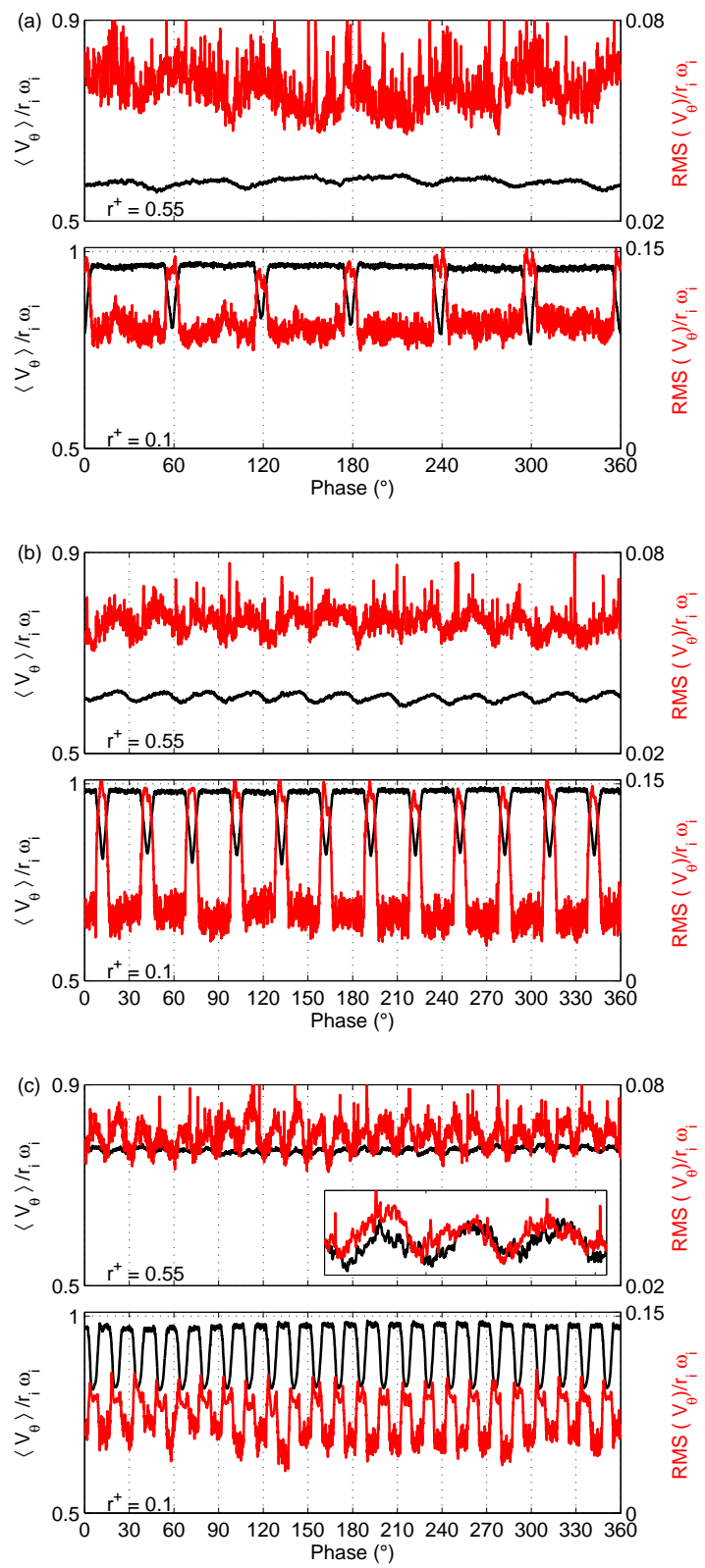

Figure 9: Normalized phase-averaged azimuthal velocity for $R e_{i}=47000$ : mean $\left\langle V_{\theta}(\phi)\right\rangle / r_{i} \omega_{i}$ (black) and root mean squared $R M S\left\langle V_{\theta}(\phi)\right\rangle / r_{i} \omega_{i}$ (red).(a) $G 6$ case, (b) $G 12$ case, and (c) $G 24$ case. The phase-averaged technique is applied only for the two locations where the spectrum density displays at least one discernible peak

\subsection{Torque}

The torque of the four inner cylinders expressed in terms of friction coefficient $C_{f}$ (see equation 2 is shown in Fig. 10 as a function of the Reynolds number $R e_{i}$. In this figure we also show the local scaling exponent $\alpha$,defined by $C_{f} \propto R e_{i}^{\alpha-2}$ and estimated as $\alpha=2+\frac{d \log \left(C_{f}\right)}{d \log \left(R e_{i}\right)}$. The derivative is computed by a convolution product of the data series with the derivative of a gaussian of length 21 points and standard deviation 11 points. Before we discuss the variation of the friction coefficient with the groove number, we compare our smooth cylinder data to the experimental results of Ravelet et al. [17, Motozawa et al. [7] and Lewis \& Swinney [20] and, to an empirical correlation of Dubrulle \& Hersant [21. The large gap between the present smooth data and those of literature is not surprising since rigorous direct comparison could not be achieved owing to the different gap ratios of the experiments and the end effects. It should be noted however that the torque is measured in the same way in Refs. [17, 7] and in the current study. The torque is moreover corrected from end effects by dividing by 2 in Ref. [17] and by using the correlation of Hudson \& Eibeck 15 in the present study. No end effects correction is used in Refs. [7, 20] since Lewis \& Swinney 20 measured the torque acting only on the central part of the inner cylinder. To obtain good agreement with the correlation of Dubrulle \& Hersant 21] rewritten as

$$
C_{f}=\frac{K_{7}}{2 \pi} \frac{(1-\eta)^{1 / 2}}{\ln \left[\eta^{2}(1-\eta) R e_{i}^{2} / K_{8}\right]^{3 / 2}},
$$

we use $K_{7}=1.95$ and $K_{8}=10^{0}$ which should be compared to the given values of $K_{7}=0.5$ and $K_{8}=10^{4}$. It should be recalled that Dubrulle \& Hersant 21] contructed

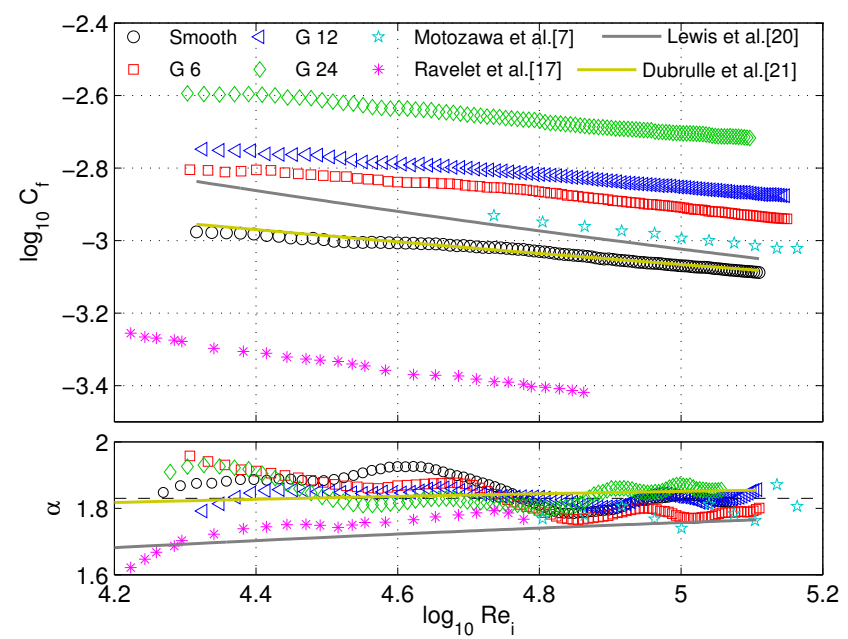

Figure 10: Friction coefficient $C_{f}$ and local exponent $\alpha$ as a function of the Reynolds number $R e_{i}$. Comparison to the experimental data of Ravelet et al. [17] $(\eta=0.917)$, Motozawa et al. [7] $(\eta=0.939)$ and Lewis and Swinney [20] (equation 3, $\eta=0.724$ ), and the empirical correlation of Dubrulle and Hersant 21 (equation 7 fitted to our data with $K_{7}=1.95$ and $K_{8}=10^{0}$ 
their correlation using results of Lewis \& Swinney 20] obtained with $\eta=0.724$.

We now analyse the influence of the groove number on the friction coefficient in relation with the Reynolds number. As indicated in Fig. 10 the friction coefficient increases with increasing groove number. Their profiles appear to be quite similar in shape without discernible transitional regime, which means that the flow is at fully developed turbulence state over the range of Reynolds numbers examined. The local scaling exponent (see bottom Fig. 10) also confirms this. The exponent for all the three grooved cylinders collapse with the smooth cylinder exponent, with mean value of $1.83 \pm 0.1$. This value agrees well with the results of Lathrop et al. [22, Lewis \& Swinney [20] and Ravelet et al. [17. In the previours experimental studies of $C_{f} \propto R e_{i}^{\alpha-2}$ (Cadot et al. [5], van den Berg et al. [23]), it was found that the value of the exponent $\alpha$ was equal to 1.88 with an inner cylinder roughened by parallel regular square cross section ribs and outer smooth cylinder, and $\alpha=2$ when both walls were rough. However our inner grooved cylinder exponents were fairly close to $\alpha=1.83$, the fact that $\alpha$ is approximately equal to the smooth cylinder value points out that the effects of grooves on the exponent might be too weak to be observed due to the large value of $\eta$ or, that the contribution of grooves might be associated to a simple shift in pressure drag. Therefore one may conclude that the present friction coefficient scaling is not bulk flow dominant contrary to the common behaviour of classical rough wall.

To further illustrate the effect of groove number on the torque, Fig. 11 presents the ratio of friction coefficients between the grooved and smooth cylinders $C_{f}^{*}=$

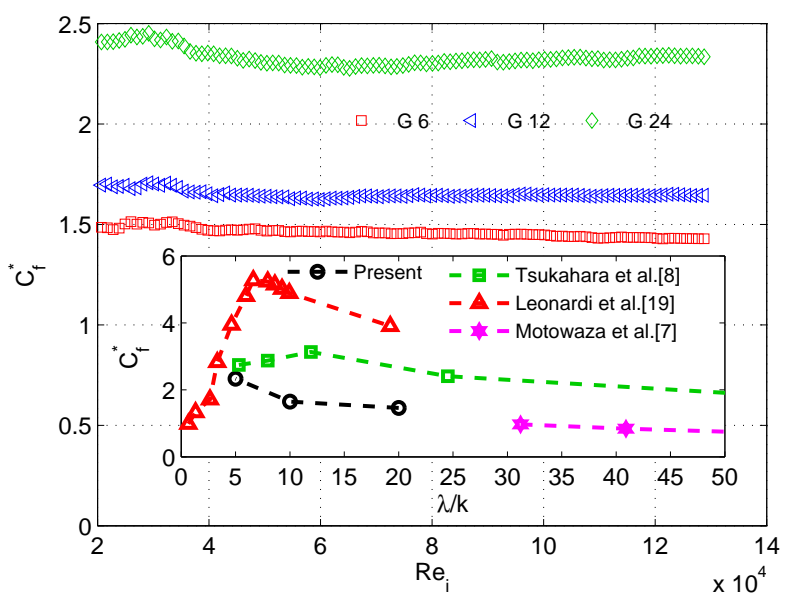

Figure 11: friction coefficient ratios between the grooved and smooth cylinders $C_{f}^{*}=C_{f} / C_{f, s m o o t h}$ as a function of $R e_{i}$. Insert: $C_{f}^{*}$ averaged over $R e_{i}$ as a function of rough element interval normalized by its height $\lambda / k$. Comparison to, DNS study of turbulent channels flow with transverse square ribs of leonardi et al. [19], experimental and DNS results of Taylor-Couette flow with triangular and square longitudinal ribs of Motozawa et al. [7] and Tsukahara et al. [8], respectively

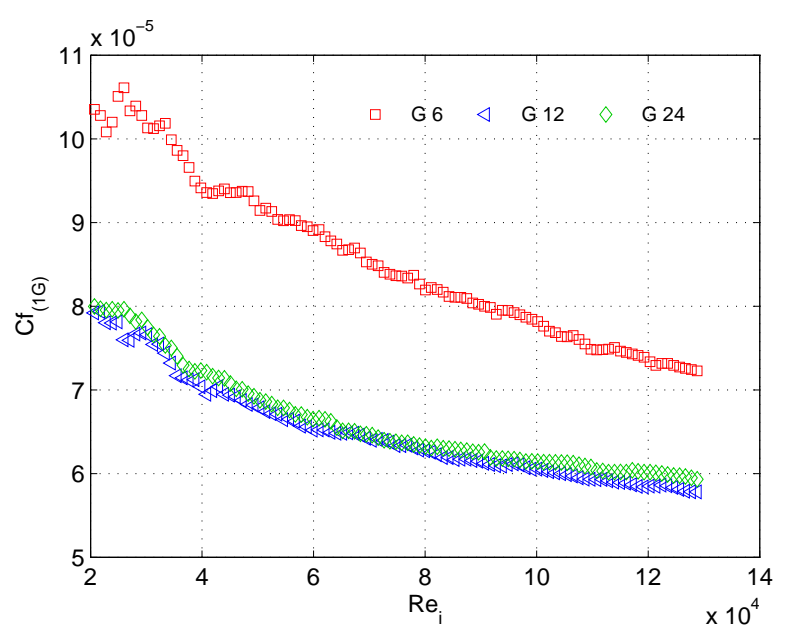

Figure 12: Relative contribution of single groove $C_{f, 1 G}$ as a function of $R e_{i}$

$C_{f} / C_{f, s m o o t h}$ as a function of $R e_{i}$. The friction ratio is approximately constant across the whole Reynolds number range. Averaged over the whole $R e_{i}$ range, the mean ratio $C_{f}^{*}$ is approximately $1.46,1.65$ and 2.33 for $G 6, G 12$, and G24, respectively. In Fig. 11, the subfigure compares the mean ratios $C_{f}^{*}$ to those of literature [19, 7, 8] as a function of the rough element interval normalized by its height $\lambda / k$. Significant differences can be observed. The grooved cylinder values are lower on average than the ribbed channel and cylinder results for all $\lambda / k$ and the peak is also shifted towards lower $\lambda / k$. In view of the present results, we can assume that the higher friction coefficient ratio reported in Refs. [19, 7, 8] can be attributed to an enhanced pressure drag by rib. Indeed, for these studies, the main flow direction is obstructed by the ribs. As a result, a strong pressure gradient is generated leading to intense transfer of momentum from fluid to the wall.

To go further in the analysis, effort is made to estimate the relative contribution of each groove by mean of two assumptions: $(i)$ the groove (cavity) contribution to the skin friction is considered negligible compared to that of the crest; ( $i i$ ) the skin friction on the crest is equall to that of smooth cylinder over the Reynolds number range. Under these two assumptions, the contribution of a single groove is defined as follows:

$$
C_{f, 1 G}=\frac{C_{f}-\frac{S_{G n}}{S_{s m o o t h}} C_{f, s m o o t h}}{n},
$$

where $S_{\text {smooth }}$ is the lateral surface area of the smooth cylinder and $S_{G n}$ is the total crest surface area of the $G_{n}$ cylinder. The contribution $C_{f, 1 G}$ can be interpreted as a single groove pressure drag contribution to the total friction coefficient. Figure 12 shows the relative contribution of single groove as a function of $R e_{i}$. The profiles are similar in shape for the three tested cylinders, even though they illustrate a substantial dependence on Reynolds number. The contribution should be equal for the three cases if 


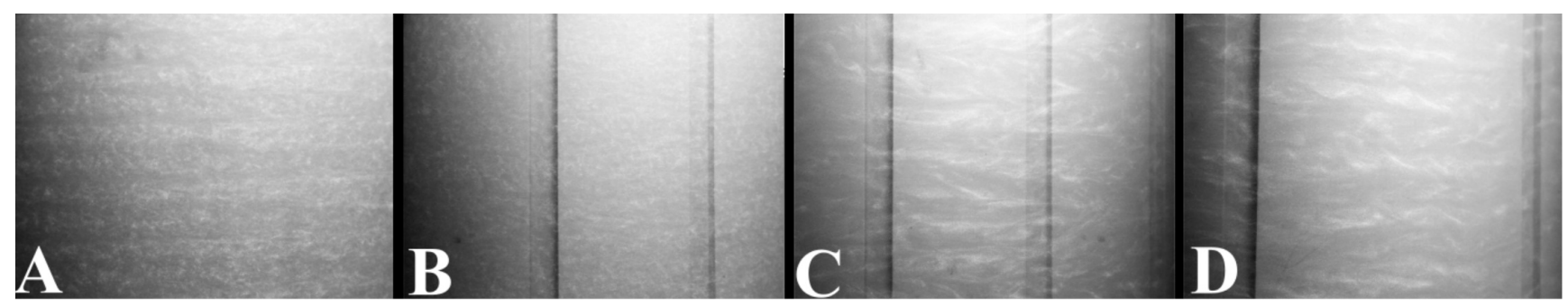

Figure 13: Photographs of the flow at different Reynolds number and surface roughness. A: smooth cylinder at $R e_{i}=4.06 \times 10^{3}$, B: $G 12$ cylinder at $R e_{i}=4.06 \times 10^{3}, \mathrm{C}: G 12$ cylinder at $R e_{i}=1.15 \times 10^{3}$,D: $G 6$ cylinder at $R e_{i}=1.15 \times 10^{3}$. The flow structure is vusualized using microscopic iriodin particles.

our assumptions are strictly valid or if there are no interaction between the groove-induced modulation. The first assumption is supported by the literature results of Hayase et al. 22 and Leonardi et al. [19] obtained with pitch ratio less than 4 which is the case of the current study $(w / k \approx 2)$. The second assumption is slightly weaker since the velocity results (see Fig. 3, 4 and 5 clearly show a steeper velocity gradient and an increase of azimuthal bulk velocity with increasing groove number. They will result in skin friction reduction on the crest through the reduction of the boundary layer contributions. The gap between G12 and G24, and $G 6$ distributions is too large to be associated only with reduction of skin friction on the crest. Based on these observations and arguments, we conclude that $G 12$ and $G 24$ flows have similar structural behaviour, strictly different from that of $G 6$, which we associate to groove density. The azimuthal velocity spectra (see Fig. 7, 8) also confirm the difference in the flow feature since the groove signature is found to persist until the near wall of the outer cylinder for $G 6$, which is not the case for $G 12$ and $G 24$. This agrees well with the finding of Tsukahara et al. [8] who observe that, for $\lambda / k \geq 13$, the rough elements are isolated. Globally, similar behaviour is reported by Motozawa et al. [7] for ribbed inner cylinder, which the authors attributes to the recovery of the velocity distribution which could not be achieved for smaller value of $\lambda / k$.

We therefore conclude that, for this type of flow configuration where surface morphology significantly acts on the global transport properties represented by measured torque, at least spatial distribution of velocity field at vicinity of wall should be derived from more complete experiments, to account for the separate effects of elementary rough element on skin friction and pressure drag, which is not the case of the current study.

\section{Conclusion and future work}

Derived from industrial situations, the experimental conditions led to the study of Taylor Couette flow with axially grooved rotating inner cylinder and fixed outer cylinder. With a large radius ratio and high Reynolds number up to $R e_{i}=13 \times 10^{4}$, this experiment simulates a compact electric motor behavior at high velocity speed. Torque and pointwise velocity measurements have been carried out for different tested inner cylinders to assess flow characteristics and global transport properties.

The most prominent differences in the flow features between the grooved tested cylinder cases and the smooth cylinder case are a loss of axial dependency of the timeaveraged velocity field; a steeper velocity gradient near the grooved rotating cylinders and an increase of the bulk flow azimuthal velocity with decreasing circumferentially periodic length $\lambda / k$. This parameter is found to discriminate the flow feature since it allows to characterize the periodic spatial variations in the flow within the roughness boundary and the bulk flow.

Direct consequence of these variations in the flow features is a large difference in global transport properties measured by the torque and expressed in term of friction coefficient. It is shown that the use of grooved inner cylinder increases the friction coefficient and that the groove contribution rate is a function of $\lambda / k$. Within the Reynolds number range of this study $\left(2 \times 10^{4} \leq R e_{i} \leq\right.$ $\left.13 \times 10^{4}\right)$, the friction coefficient ratio between the rough and the smooth cylinders is approximately constant and the mean ratio is approximately $1.46,1.65$ and 2.33 for $G 6, G 12$, and $G 24$, respectively. Despite these strong effects on the measured torque, the local scaling exponent of the friction factor with Reynolds number seems insensitive, contrary to the common behaviour of classical rough wall.

The current study must be only the start of a long experimental program to further explore the effects of axial rectangular groove on turbulent Taylor-Couette flow. The most immediate and urgent objective will be to perform high spatial resolution velocity measurement in the rotating grooved inner cylinder sublayers in order to estimate local contribution of skin friction and pressure drag. It should be noted that all of the experiments were conducted for a single value of pitch to height ratio $(w / k \approx 2)$. Hence, future experiment should be performed for different values of $w / k$ to confirm ours observations.

The next step will be to examine the flow dynamics and the transport properties at lower Reynolds numbers. In fact, it is interesting to observe in Fig. 13 that different turbulent states coexist. At $R e_{i}=4.06 \times 10^{3}$, the smooth cylinder flow is dominated by Taylor-vortices while the $G 12$ flow did not contain obvious structures like 
Taylor-vortices. By decreasing the Reynolds number from $R e_{i}=4.06 \times 10^{3}$ to $R e_{i}=1.15 \times 10^{3}$, a bifurcation process occurrs for $G 12$ flow (see Fig. $13 \mathrm{C}$ ) revealed by the presence of large coherence structures with azimuthal wavelengths locked to $\lambda$. This is confirmed by the Photograph in Fig. 13 D obtained with G6. This kind of study has been performed by Ravelet et al. 24] in inertially driven von Kármán closed flow in which the authors observed the so-called 'turbulent bifurcation' and multistability. The objective will be to check whether these flow features and bufircation are reflected in the overall transport properties.

\section{References}

[1] M. Fnot, Y. Bertin, E. Dorignac, G. Lalizel, A review of heat transfer between concentric rotating cylinders with or without axial flow, International Journal of Thermal Sciences 50 (2011) 1138-1155. doi:10.1016/j.ijthermalsci.2011.02.013

[2] T. Hayase, J. A. C. Humphrey, R. Greif, Numerical calculation of convective heat transfer between rotating coaxial cylinders with periodically embedded cavities, Journal of Heat Transfer 114 (1992) 589-597. doi:10.1115/1.2911322

[3] Y. N. Lee, W. Minkowycz, Heat transfer characteristics of the annulus of twocoaxial cylinders with one cylinder rotating, International Journal of Heat and Mass Transfer 32 (1989) 711722. doi:10.1016/0017-9310(89)90218-4

[4] S. Gardiner, R. Sabersky, Heat transfer in an annular gap, International Journal of Heat and Mass Transfer 21 (1978) 14591466. doi:10.1016/0017-9310(78)90002-9

[5] O. Cadot, Y. Couder, A. Daerr, S. Douady, A. Tsinober, Energy injection in closed turbulent flows: Stirring through boundary layers versus inertial stirring, Physical Review E 56 (1997) 427433. doi:10.1103/PhysRevE.56.427

[6] T. H. van den Berg, C. R. Doering, D. Lohse, D. P. Lathrop, Smooth and rough boundaries in turbulent taylor-couette flow, Physical Review E 68 (2003) 036307. doi:10.1103/PhysRevE. 68.036307

[7] M. Motozawa, T. Ito, K. Iwamoto, H. Kawashima, H. Ando, T. Senda, Y. Tsuji, Y. Kawaguchi, Experimental investigations on frictional resistance and velocity distribution of rough wall with regularly distributed triangular ribs, International Journal of Heat and Fluid Flow 41 (2013) 112-121. doi:10.1016/j. ijheatfluidflow.2013.03.004

[8] T. Tsukahara, M. Ishikawa, Y. Kawaguchi, Dns study of the turbulent taylor-vortex flow on a ribbed inner cylinder, Advances in Mechanical Engineering (2013) 628490doi:10.1155/ 2013/628490

[9] T. Hall, D. Joseph, Rotating cylinder drag balance with application to riblets, Experiments in Fluids 29 (2000) 215-227. doi:10.1007/s003489900075.

[10] H. V. Moradi, J. M. Floryan, Flows in annuli with longitudinal grooves, Journal of Fluid Mechanics 716 (2013) 280-315. doi: 10.1017/jfm.2012.547

[11] A. J. Greidanus, R. Delfos, S. Tokgoz, J. Westerweel, Turbulent taylor-couette flow over riblets: drag reduction and the effect of bulk fluid rotation, Experiments in Fluids 56 (2015) 107. doi:10.1007/s00348-015-1978-7.

[12] X. Zhu, R. Ostilla-Mnico, R. Verzicco, D. Lohse, Direct numerical simulation of taylorcouette flow with grooved walls: torque scaling and flow structure, Journal of Fluid Mechanics 794 (2016) 746-774. doi:10.1017/jfm.2016.179

[13] M. Couette, Etude sur le frottement des liquids, Annales de chimie et de physique 21 (1890) 433-510.

[14] S. Grossmann, D. Lohse, C. Sun, High reynolds number taylorcouette flow, Annual Review of Fluid Mechanics 48 (2016) 53-80.

[15] A. J. Hudson, P. A. Eibeck, Torque measurements of corotating disks in an axisymmetric enclosure, Journal of Fluids Engineering 113 (1991) 648-653. doi:10.1115/1.2926529

[16] S. G. Huisman, D. P. van Gils, C. Sun, Applying laser doppler anemometry inside a taylor-couette geometry using a ray-tracer to correct for curvature effects, European Journal of Mechanics - B/Fluids 36 (2012) 115-119. doi:10.1016/j.euromechflu. 2012.03.013

[17] F. Ravelet, R. Delfos, J. Westerweel, Influence of global rotation and reynolds number on the large-scale features of a turbulent taylorcouette flow, Physics of Fluids 22 (2010) 055103. doi: 10.1063/1.3392773

[18] A. E. Perry, W. H. Schofield, P. N. Joubert, Rough wall turbulent boundary layers, Journal of Fluid Mechanics 37 (2) (1969) 383-413. doi:10.1017/S0022112069000619.

[19] S. Leonardi, P. Orlandi, R. J. Smalley, L. Djenidi, R. A. Antonia, Direct numerical simulations of turbulent channel flow with transverse square bars on one wall, Journal of Fluid Mechanics 491 (2003) 229-238. doi:10.1017/S0022112003005500

[20] G. S. Lewis, H. L. Swinney, Velocity structure functions, scaling, and transitions in high-reynolds-number couette-taylor flow, Physical Review E 59 (1999) 5457-5467. doi:10.1103/ PhysRevE.59.5457

[21] B. Dubrulle, F. Hersant, Momentum transport and torque scaling in taylor-couette flow from an analogy with turbulent convection, The European Physical Journal B - Condensed Matter and Complex Systems 26 (3) (2002) 379-386. doi:10.1140/ epjb/e20020103

[22] D. P. Lathrop, J. Fineberg, H. L. Swinney, Transition to sheardriven turbulence in couette-taylor flow, Physical Review A 46 (1992) 6390-6405. doi:10.1103/PhysRevA.46.6390.

[23] T. H. van den Berg, C. R. Doering, D. Lohse, D. P. Lathrop, Smooth and rough boundaries in turbulent taylor-couette flow, Physical Review E 68 (2003) 036307. doi:10.1103/PhysRevE. 68.036307

[24] F. Ravelet, A. Chiffaudel, F. Daviaud, Supercritical transition to turbulence in an inertially driven von kármán closed flow, Journal of Fluid Mechanics 601 (2008) 339-364. doi:10.1017/ S0022112008000712 